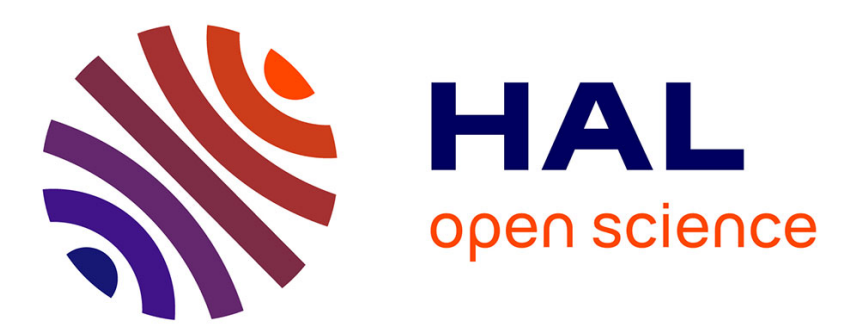

\title{
Interaction Techniques for Visual Exploration Using Embedded Word-Scale Visualizations
}

\author{
Pascal Goffin, Petra Isenberg, Tanja Blascheck, Wesley Willett
}

\section{To cite this version:}

Pascal Goffin, Petra Isenberg, Tanja Blascheck, Wesley Willett. Interaction Techniques for Visual Exploration Using Embedded Word-Scale Visualizations. Conference on Human Factors in Computing Systems (CHI), Apr 2020, New York, NY, United States. 10.1145/3313831.3376842 . hal-02459518

\author{
HAL Id: hal-02459518 \\ https://hal.inria.fr/hal-02459518
}

Submitted on 29 Jan 2020

HAL is a multi-disciplinary open access archive for the deposit and dissemination of scientific research documents, whether they are published or not. The documents may come from teaching and research institutions in France or abroad, or from public or private research centers.
L'archive ouverte pluridisciplinaire HAL, est destinée au dépôt et à la diffusion de documents scientifiques de niveau recherche, publiés ou non, émanant des établissements d'enseignement et de recherche français ou étrangers, des laboratoires publics ou privés. 


\section{Interaction Techniques for Visual Exploration Using Embedded Word-Scale Visualizations}

\author{
Pascal Goffin \\ University of Utah \\ Salt Lake City, USA \\ ppjgoffin@gmail.com
}

\author{
Tanja Blascheck \\ University of Stuttgart \\ Stuttgart, Germany \\ research@blascheck.eu
}

\author{
Petra Isenberg \\ Université Paris-Saclay, \\ CNRS, Inria, LRI \\ 91405 Orsay, France \\ petra.isenberg@inria.fr
}

\author{
Wesley Willett \\ University of Calgary \\ Calgary, Canada \\ wj@wjwillett.net
}

\begin{abstract}
We describe a design space of view manipulation interactions for small data-driven contextual visualizations (word-scale visualizations). These interaction techniques support an active reading experience and engage readers through exploration of embedded visualizations whose placement and content connect them to specific terms in a document. A reader could, for example, use our proposed interaction techniques to explore word-scale visualizations of stock market trends for companies listed in a market overview article. When readers wish to engage more deeply with the data, they can collect, arrange, compare, and navigate the document using the embedded word-scale visualizations, permitting more visualizationcentric analyses. We support our design space with a concrete implementation, illustrate it with examples from three application domains, and report results from two experiments. The experiments show how view manipulation interactions helped readers examine embedded visualizations more quickly and with less scrolling and yielded qualitative feedback on usability and future opportunities.
\end{abstract}

\section{Author Keywords}

Information visualization; word-scale visualization; interaction techniques; text visualization; glyphs.

\section{CCS Concepts}

-Human-centered computing $\rightarrow$ Visualization;

\section{INTRODUCTION}

Word-scale visualizations are small text-embedded data graphics that visualize data related to individual terms (entities) in a document [33]. For example, a text with small embedded stock trends DAX detailed overview of a stock's performance, without requiring the reader to shift attention away from the text to larger visualizations or tables located elsewhere.

Researchers have used word-scale visualizations in settings such as software source code [7, 11, 35, 51], bibliometrics [39],

Permission to make digital or hard copies of all or part of this work for personal or classroom use is granted without fee provided that copies are not made or distributed for profit or commercial advantage and that copies bear this notice and the full citation on the first page. Copyrights for components of this work owned by others than the author(s) must be honored. Abstracting with credit is permitted. To copy otherwise, or republish, to post on servers or to redistribute to lists, requires prior specific permission and/or a fee. Request permissions from permissions@ acm.org.

CHI '20, April 25-30, 2020, Honolulu, HI, USA.

(C) 2020 Copyright is held by the owner/author(s). Publication rights licensed to ACM ACM ISBN 978-1-4503-6708-0/20/04 \$15.00.

http://dx.doi.org/10.1145/3313831.3376842 scientific publications [3, 12, 24], sports reports [47], and data comics [4]. Word-scale visualizations have also appeared in online news articles and websites [21] and are typically used to augment data-rich text with concise visual representations of data that would be difficult or impossible to describe using words alone. Interactive word-scale visualizations represent an opportunity to create rich reading experiences that transcend the limitations of traditional text + figure paradigms. While larger and more complex visualizations outside the text can provide other benefits including more detail and easier comparisons, we show how view manipulation interactions can also add many of these benefits to word-scale visualizations.

Most examples of word-scale visualizations in-the-wild (Figure 2) are static charts embedded at fixed locations in documents with no support for interactivity. This makes sense when text and visualizations are intended for print. Yet, today most text is consumed on personal computers such as phones, tablets, e-readers, desktops, or laptops. In these environments people can engage actively with the text and its embedded content to explore, make comparisons, and extract details.

In this paper we outline a design space of view manipulation interactions to expand the active reading capabilities of embedded word-scale visualizations. This design space, in particular, allows interactive word-scale visualizations to serve as

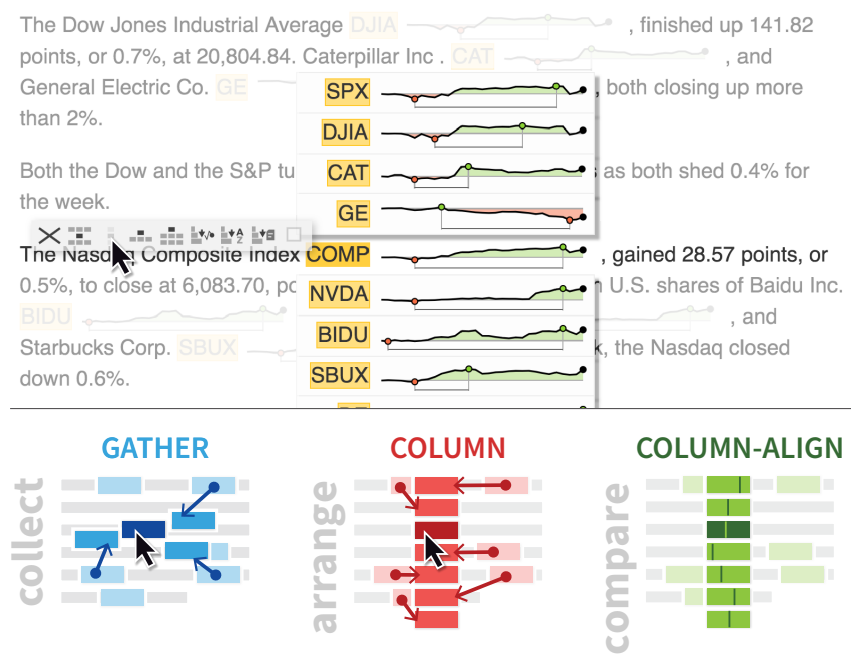

Figure 1. Word-scale stock charts in a news article (top) collected using a gather operation and arranged into a column with a column-aligned layout to support comparison. 
a bridge between document-centric and visualization-centric analyses. In document-centric analyses, the document is the main point of interest and figures provide supporting evidence or contextual information. In visualization-centric analyses, source text is a secondary element (if it is present at all) and large visualizations become the focus of the viewer's attention. Example tools that use documents as a data source while providing larger visualization environments include DocuBurst [25] or Jigsaw [50]. However, no current environment supports fluidly transitioning between document-centric analyses that include visualizations and visualization-centric analyses that still have a close connection to the text.

Our design space of view manipulation interactions bridges this gap with interaction techniques that allow readers to collect, arrange, compare, and navigate word-scale visualizations in a document (Figure 1). Dynamic transitions between reading the document and seeing larger small multiple visualizations (Figure 1-bottom) for a visualization-centric analysis become possible. To illustrate how different interactions from the design space can be combined and used, we developed an interactive prototype as an extension to the web-based Sparklificator library [33] and show how to use these view manipulation interactions with examples from economic news, the humanities, and a research report of an eye tracking study. Many instantiations of our design space are possible. We conducted two experiments that tested the impact of specific transitions between document-centric and visualization-centric analyses. In the first experiment we examine the impact of basic gathering and sorting interactions on search and comparison tasks. In the second experiment we qualitatively examine view manipulation interactions during an open-ended analysis. We found that view manipulation interactions were well-received and helped readers perform comparison tasks faster and more accurately with less scrolling.

\section{RELATED WORK}

Our research takes inspiration from work on interactive text analysis, previous work on the general concept of small contextual visualizations, and past work that has discussed possible interactions for word-scale visualizations.

\section{Interactive Text Analysis}

Several interactive text analysis tools integrate text and data. Systems like Popout Prism [52] and VisRa [43] aid document analysis by using color, size and other visual attributes to encode information about readability, sentiment, and important entities within the text. In contrast to these systems, other work has explored encoding information outside of a document and using graphical methods to reconnect visualizations and text. For example, Steinberger et al. [51] connect entities to marks in nearby visualizations by drawing "contextpreserving" graphical links. Similarly, Kong et al. [38] establish a text-to-graphic relationship through interactive brushing of text and highlighting of corresponding marks in a visualization. Boy et al. [18] discuss in more detail how "suggested interactivity" cues added to embedded visualizations in documents imply interactivity and convey the possibility of data exploration. Like these previous works, we focus on documents with embedded data, yet at a smaller word-scale. We

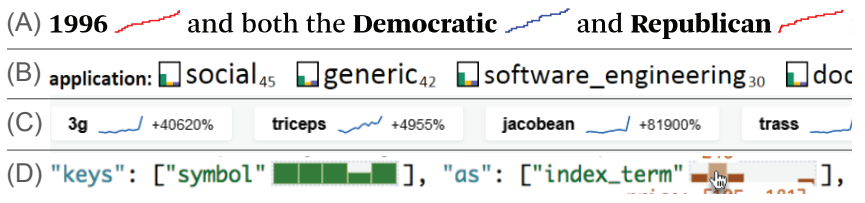

Figure 2. Examples of word-scale visualization use in the wild: (A) in a political news article from FiveThirtyEight [21], (B) embedded inline in the SurVis analysis tool [10], (C) a word trend ticker on dictionary.com [2], and (D) inline in Hoffswell et al.'s [36] source code editor.

also employ interaction to connect text and data graphics but support fluid ad-hoc switching between reading text and exploring the embedded visualizations.

Our design space of view manipulation interactions is related to a variety of other tools that help analysts interactively organize and compare elements within a text or a visualization. Victor's "explorable explanations" [59] show how to use direct manipulation of text or visual elements to highlight relationships resulting in an improved active reading experience. In many of his examples, interacting with one element changes other elements based on a previously established relationship. Closely related are Dragicevic et al.'s [27] explorable multiverse analysis reports (EMAR) which introduce interactive graphics into the results sections of research articles. In contrast to this work, we focus on interactions specifically for multiple small-scale visualizations and not on in-text controls for modifying the parameters of larger visualizations.

Several past interaction techniques have directly influenced our design space. Fluid Documents [23, 64], for example, describes techniques to display annotations for entities. For instance, such documents allow the expansion and typographic shrinking of text and provide ways to move the annotations away from the text, such as into the margin. This work influences the no-overlap layout placement we discuss in our design space. We also discuss a technique similar to the "Bring \& Go" interaction technique [42], which was originally devised to support the exploration of network diagrams by pulling connected nodes closer together. In addition, we take inspiration from GraphDice [16] and Small MultiPiles [5], which provide interactive tools for organizing and comparing scatter plot matrices and small multiples. These approaches motivated our approaches to how multiple word-scale visualizations are ordered in a structured layout for more meaningful comparisons.

\section{Small Contextual Visualizations}

Small contextual data-driven visualizations have been discussed under several names, such as sparklines [56], wordscale visualizations [33], and micro visualizations [44, 45]. Tufte introduced sparklines as "small, intense, simple, wordsized graphics with typographic resolution." His examples focus primarily on printed text and do not specifically mention interaction. Parnow and Dörk defined micro visualizations as data representations that are small in physical display space, used in the context of documents, and encode few data dimensions. Meanwhile, Goffin et al. [33, 32] use "word-scale visualization" to describe a broader set of small-scale graphics that are larger than the typographic resolution of sparklines and have more data dimensions than micro visualizations. Brath et al. [20] introduced a design space for SparkWords, a related 
concept which embeds categorical, ordered, or quantitative data into letters or words by modifying their typography, color, and other properties. More recently, Latif and Beck [39] introduced a design space for word-sized graphics based on the data visualized and show that even multivariate, spatial, and relational data can be represented at a small scale. Researchers and designers have also proposed static word-scale visualizations for a variety of specific applications including word-sized eye tracking visualizations [9], Gestaltlines [19] which leverage gestalt properties to encode multivariate data, and SportLines [47] which show key phases in soccer matches.

\section{Interactive Word-Scale Visualizations}

Previous research has also introduced several kinds of interaction techniques for word-scale visualizations. Parnow [45] describes techniques for placing micro visualizations into the text, brushing and linking between micro visualizations, and highlighting connections between entities and visualizations. We incorporate a similar brushing and linking approach into our design space. Meanwhile, Beck and Weiskopf [12] propose three levels of interaction (no interaction, local interaction, and global interaction) for word-sized graphics. Their global interaction level, which includes interactions between multiple charts comes closest to the spirit of our design space. However, we focus on interactions for manipulating and organizing word-scale visualizations into small multiples arrangements, which are not discussed in their work.

As a result of an exploratory study with designers, Goffin et al. [34] highlight several potential interaction techniques for word-scale visualizations. In their study designers suggested interactions to connect, compare, filter, and highlight multiple word-scale visualizations. They also proposed interactions for incorporating word-scale visualizations on-demand and switching between alternative views of the same data. Moreover, Goffin et al.'s interaction design space describes opportunities for interacting with individual word-scale visualizations, including possible UI elements and interaction scopes. We elaborate on several of these proposed interaction techniques in our design space for view manipulation interactions.

Several concrete examples of interactive word-scale visualizations also exist in other domains. To assist in debugging electronic circuits Frishberg [29] proposed interactions for details-on-demand by clicking on a data item in the sparkline. Similarly, Watts [61] implemented a jQuery library for dynamic and interactive sparklines that allows hovering over data items to provide more information. Meanwhile, Hoffswell et al. [36] describe and implemented a design space of word-scale visualizations for source code. Their designs demonstrate several interactions, including hovering to show details-on-demand as well as a variant of brushing and linking.

Finally, Latif et al. [41] describe a framework that integrates text, word-scale visualizations, and larger visualizations. In their framing, each element has triggers and filters to provide details-on-demand, highlight content, reset a visualization, or switch its content. Latif and Beck [40] extend this approach in VIS Author Profiles a visual analytics system for examining research profiles. They provide a tighter connection between word-scale visualizations and larger visualizations, including interactions that overlay word-scale visualizations onto larger charts. In contrast, we focus primarily on view manipulation interactions for larger numbers of word-scale visualizations.

\section{A DESIGN SPACE OF VIEW MANIPULATION INTERACTIONS FOR WORD-SCALE VISUALIZATIONS}

View Manipulation [35] tasks are vital to visualization scenarios in which elements need to be selected, navigated, coordinated, and organized. Word-scale visualizations are elements that could benefit from interactive organization and comparison while keeping a close tie to the source text. Our work explores view manipulation interactions to support richer active reading and data exploration with word-scale visualizations. We organize them into a design space of view manipulation interactions for small data representations. In particular, we focus our design space around four types of analysis tasks:

T1: Collecting word-scale visualizations. Creating small multiple visualizations that bring together visualizations from across the document for higher-level analyses.

T2: Arranging word-scale visualizations. Organizing visualizations to expose trends and support reasoning.

T3: Comparing word-scale visualizations. Providing tools to make direct comparisons between visualizations.

T4: Navigating the document. Using visualizations as entry points to the text to aid navigation to related locations.

We explored the design space through iterative rounds of ideation, sketching, and prototyping. We began by examining each of the general classes of interactions above, then worked to describe, categorize, and test a diverse set of interactions by which each could be carried out.

\section{Collecting Word-Scale Visualizations}

When reading long scrollable documents enriched with multiple word-scale visualizations it may be difficult to obtain a clear enough overview of the visualizations to analyze the underlying data. This is especially true if the visualizations are embedded throughout the document and impossible to view simultaneously on one screen. However, seeing an overview is often an important first step before engaging in other data exploration tasks such as arranging, comparing, sorting, and navigating the data. To support full or partial overviews, we propose two interaction techniques for collecting word-scale visualizations from across a document: gather and drag.
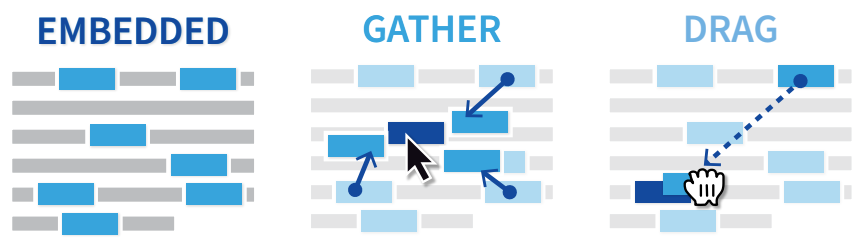

The gather interaction, inspired by Moscovitch et al.'s [42] "Bring \& Go" and Ghani et al.'s [30] "Dynamic Insets" allows a reader to quickly collect a set of word-scale visualizations at one specified location in the document. Using this interaction, a reader summons some or all of the visualizations in the text, making it possible to examine them simultaneously. When gathered, visualizations animate from their original distributed positions in the document to a central location. While this point could fall anywhere on or off-screen, we suggest gathering visualizations around a specific entity of interest, which 
we call a focus entity. This permits readers who are focused on the text to gather multiple visualizations at their current reading position, allowing them to continue reading while still using the gathered visualizations for context and comparison. In cases in which a reader only wishes to compare a small number of visualizations, dragging individual charts together provides a simpler and more predictable collection method.

Both gathering and dragging require design decisions about several different aspects of these two interactions:

Multiple encodings/semantics in document. A document may contain word-scale visualizations showing different datasets which may or may not be comparable to one another. These initial conditions should be considered when gathering or dragging word-scale visualizations. In these cases, readers may wish to only gather those visualizations which share the same data types, encodings, or semantics. Alternatively, a tool could gather each type of word-scale visualization into its own distinct cluster.

Scaling. To support meaningful comparisons between multiple word-scale visualizations which display the same kind of data, all visualizations must use the same layout, size, and color scales. However, using a shared scale for all word-scale visualizations may also hide important details in individual charts. Dynamically aligning the scales for multiple charts when they are collected together can help ensure that they are comparable, but may also entail complex transitions.

Connecting to previous locations. When gathering wordscale visualizations or dragging a word-scale visualization to another place in a document, it is important to consider how and if to fill the original location of the dragged item. Completely removing the word-scale visualizations leaves gaps in the text. As a result, we find it helpful to leave a copy of the entity and visualization at the original position in the document. We call this copy a ghosted word-scale visualization as it keeps a visually de-emphasized copy of the original element in the text, provides provenance information, and indicates that a copy of the word-scale visualization is currently being shown elsewhere. Leaving the ghosted visualizations in place also prevents the text from re-flowing and preserves the document layout.

Dragging out of the viewport. A word-scale visualization may need to be dragged to a location in the document outside of the current viewport not visible to a reader. Selecting and dragging a word-scale visualization should automatically scroll the viewport when the mouse reaches the viewport's edge. Word-scale visualizations can also have suggested interactivity cues [18] to let the reader know whether or not a word-scale visualization can be dragged to a given location.

Retaining connections to entities. To preserve the connection between the text and the visualizations when gathering or dragging, word-scale visualizations can be graphically coupled to the text entities to which the data refers (such as a name, a place, or a stock symbol SPX small snippets of entity text can then serve as labels that identify the charts and preserve a semantic link back to the document when word-scale visualizations are collected. In addition, rendering traces that connect gathered entities to their original positions (as in Figure 3-bottom right) further reinforces visualization-to-document relationships.

Duplicate word-scale visualizations. A document can include multiple occurrences of the same entity and duplicate word-scale visualizations. One option to avoid multiple copies in gathered overviews is to explicitly disallow duplicates and render only one word-scale visualization per entity. However, designers must then choose which instance of the entity to annotate with a word-scale visualization. In documents that include duplicate word-scale visualizations, designers can combat redundancy, for example, by visually stacking duplicates on top of each other when gathered and adding branching traces that link charts back to all original locations in the document.

Release back to original locations. When a reader has finished their comparison, the collected word-scale visualizations need to be released back to their original positions. Using animated transitions to move charts back to their original positions can reinforce where the word-scale visualizations came from and help signal their distribution in a document.

\section{Organizing Word-Scale Visualizations}

Collecting word-scale visualizations around a focus entity using a gather interaction helps to provide an overview of the visualizations but does not support more detailed comparisons. To see trends or differences more clearly, the gathered word-scale visualizations need to be arranged into layouts that convey order and support examination.

\section{Layouts}

As Beck and Weiskopf [12] highlight, existing uses of wordscale visualizations often tend to place them in tables, lists, or other small multiples layouts [55]. Once a reader collects visualizations, the choice of layout determines how many word-scale visualizations can be viewed simultaneously as well as how readers can examine and compare them.
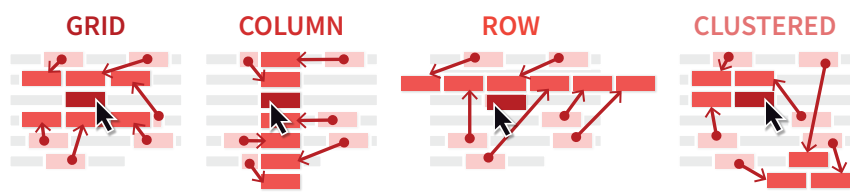

Grid layouts make good use of available screen space by placing visualizations both vertically and horizontally. However, they make comparisons across rows or columns difficult. Column layouts and row layouts each support consistent comparisons along the charts' principle axes, but permit fewer visualizations on screen. Column layouts support comparison along the charts' $x$-axis, but may require considerable scrolling and can obscure large sections of the document. Row layouts, meanwhile, may obscure as little as one line of text, but are limited by the relatively narrow width of most documents.

While few current applications use non-rectilinear layouts for word-scale visualizations [12], they may be useful in some scenarios. For example clustered layouts, which organize entities based on common characteristics could support identification and comparison of groups of charts.

In any layout, the number of visible word-scale visualizations is limited by screen space. If there are more visualizations than 
fit the screen, readers can use standard navigation techniques such as scrolling to see them or zooming out to an overview. Also, interactive techniques such as sorting, filtering, or selective gathering can help readers when a larger number of small multiples are present.

\section{Layout Placement}

Once a layout has been chosen, it needs to be placed relative to the document, entailing a number of further design decisions.

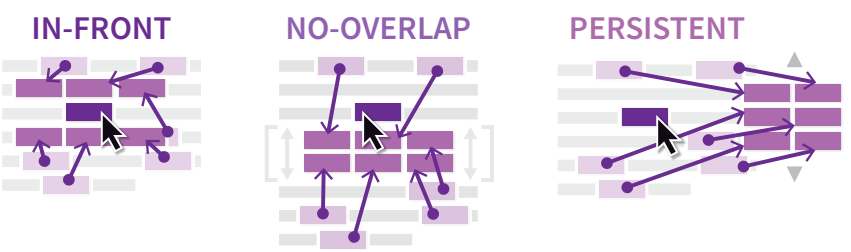

Being able to quickly glance at the text when analyzing gathered word-scale visualizations can provide clues that help contextualize the data. Placing a layout in-front of the text in a separate layer obscures some text, but keeps the layout of the document stable. Alternatively, embedding rectilinear layouts like grids and rows into the text can require re-flowing the document to create the necessary space-similar to Yoon et al.'s approach for placing ink annotations [63]. These no-overlap placements avoid collisions between the visualizations and text and guarantee that the layout is placed close to a focus entity. However, by introducing additional space, no-overlap placements also change the overall size of the document.

Applications may also use persistent placements that position the collected word-scale visualizations at a fixed location on the page and allow the document to be scrolled independently of the layout. This can make it easier to use the word-scale visualizations as an index to the document and help readers skim the document without losing track of the collected visualizations. However, it may also increase the distance between the layout and visualizations' original positions in the document.

\section{Filtering}

In documents with large numbers of word-scale visualizations, readers may want to see only a subset at any given time. Interactive filtering controls allow readers to dynamically hide and show visualizations based on properties of the data, their associated entities or their semantics. For example, an article with many embedded charts could be tailored to show a limited number of visualizations per paragraph, to show only visualizations linked to specific entities, or with particular semantics. Alternatively, a reader could gather word-scale visualizations to obtain an overview of the document, then filter the overview to examine and compare the most relevant subset.

\section{Ordering}

Interactively reordering word-scale visualizations within a gathered layout can make it easier for readers to see trends and relationships within groups of visualizations.
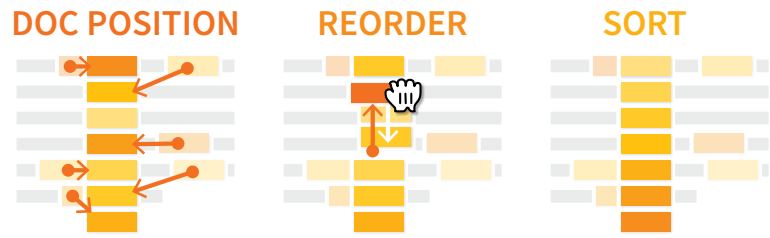

Ordering visualizations based on their document position provides structural consistency between the layout and the text as word-scale visualizations appear in the same order in both places. Alternatively, manually reordering allows readers to impose their own structure by grouping related visualizations together. Meanwhile, sorting visualizations based on their data values (max, min, average), similarity, or properties of their entities (name, date, etc.), can reveal relationships between visualizations that exist far apart in the document.

\section{Comparing Word-Scale Visualizations}

Word-scale visualizations in an ordered or sorted layout are already much easier to compare through juxtaposition [31] than word-scale visualizations scattered across a document. However, fine-grained comparisons, such as comparing values between charts with different axes is still difficult. We describe two techniques which support more detailed comparisons between word-scale visualizations-interactions for aligning grouped charts and interactions for directly comparing values.

\section{Aligning}

In row and column layouts, word-scale visualizations can be aligned in various ways. We present three alignment operators that are useful for different data comparisons. We focus on cases in which the document contains comparable word-scale visualizations. However, if a document contains multiple types of charts or data which cannot be compared directly (such as stock prices and employment figures) it may be necessary to group related visualizations before aligning them.
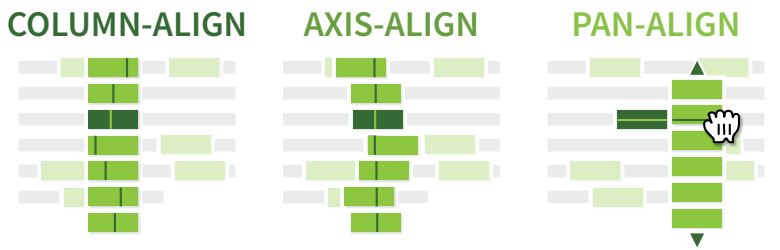

The first interaction left-aligns the word-scale visualizations in a column (column-align) and allows readers to vertically compare across the $x$-axis. However, if the $x$-axes of different visualizations are shifted relative to one another an axis-alignment may be more useful. For example, when comparing timelines that have different start and end dates, axis-alignment chooses a common date as the alignment's center. Interactive (panalign) techniques can also be applied when the word-scale visualizations are arranged in a column or row adjacent to a focused chart. In this case, panning the column or row slides the word-scale visualizations of interest past the focused chart, enabling side-by-side comparisons.

\section{Direct Comparison}

While side-by-side comparison are useful, more direct comparison mechanisms are often necessary [54].
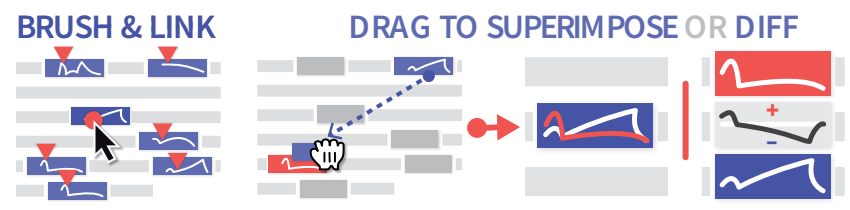

One simple technique is brushing and linking between wordscale visualizations. When a reader brushes over values in 
one word-scale visualization, corresponding values in other visualizations are highlighted [13]. Drag interactions can also be used to support comparison by either superimposing wordscale visualizations on top of one another, by triggering the creation of an explicit encoding such as difference computations between the data in multiple charts, or by juxtaposing word-scale visualizations [31].

\section{Navigating the Document}

Word-scale visualizations in documents are useful, in part, because they support ad-hoc transitions between reading a document and exploring associated data. Interactions that allow a reader to jump from a collected word-scale visualization back to its original position in the document supports fluid transitions between these modes. These kinds of navigation interactions exhibit two properties of Elmqvist et al.'s [28] characterization of fluid interfaces, promoting flow and enabling seamless switching between exploration and reading. NAVIGATION

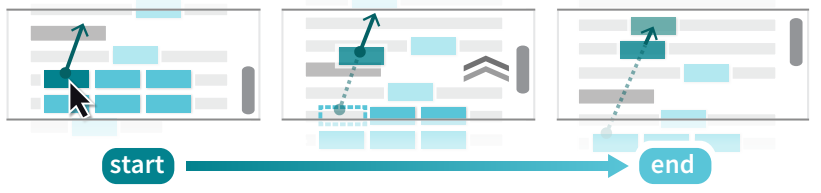

With these kinds of navigation interactions, sets of collected word-scale visualizations can serve as an index or entry point into the document, providing graphical overviews reminiscent of interfaces like Koch et al.'s "VarifocalReader" [37].

\section{PROTOTYPES}

To demonstrate how elements from this design space translate into concrete applications, we implemented an active reading environment that supports interactive word-scale visualizations and illustrate its functionality using documents from economic journalism, contemporary European history, and eye tracking research. For each context we designed or used custom word-scale visualizations.

Our economic journalism example allows readers to collect, arrange, compare, and navigate word-scale visualizations embedded in news articles extracted from MarketWatch [1]—one of many economic news outlets that already uses small stock charts in their articles (typically as tooltips over individual stock symbols or indexes). Our prototype (Figure 3-left) embeds small stock charts directly into text of articles and adds view manipulation interactions to make the charts easier to examine and compare. We use a stock chart design $\mathrm{SPX}$, which shows the performance of a single stock or financial index over the 30 days leading up to the publication of the article. The dark line on the chart shows the stock's performance relative to its value at the beginning of the 30-day period. The light grey horizontal baseline shows the price on the first day. If the price on a given day was lower than the price at the beginning of the 30-day period, the area between the line and the baseline is colored red. If the price was higher, the area is colored green. A red dot shows the day with the lowest price, while a green dot shows the day with the highest price. A grey horizontal line highlights distance between the lowest and highest days.
In our second example, we embed timeline charts into articles on European history to help readers better understand the temporal relationships and overlaps between historical figures, movements, and events (Figure 3-top right). We use a simple timeline design Alexandra Fedorovna $\_$with fixed start and end dates that visualizes lifespans and periods using blue highlights. Viewers can use the timelines to quickly check for overlaps between events and the lifespans of historical figures, making them easier to contextualize and compare.

For the third example, we created an interactive version of an academic research report, which incorporates word-scale eye tracking visualizations by Beck et al. [8] (Figure 3-bottom right). Throughout the document we visualize eye movement data using Beck et al.'s gridded attention maps 드, each of which shows the spatial distribution of visual fixations for one participant during an experimental trial. The cells with the most fixations appear dark, while those with the least appear white. Viewers can use grouping and sorting operations to more easily compare the performance of multiple participants, and identify ones who used similar strategies.

To minimize distracting overlaps between word-scale visualizations and text and help preserve readability and context, all three examples used in-front and no-overlap layout placements when gathering charts. The in-front placement always keeps the line of text containing the focus entity visible, while the no-overlap placement keeps all text visible by gathering charts between paragraphs.

Our prototype extends the Sparklificator library [33], which allows developers to insert a variety of word-scale visualizations into HTML documents. We implemented visualizations using JavaScript and D3.js. The source code and the three working examples of our prototype are available at https://github.com/InteractionWSV. To use this library, the entities in an HTML text must be tagged with span tags and should use a distinctive class name. Developers can use existing word-scale visualization designs or implement their own word-scale visualization renderers.

\section{STUDY}

While many examples of static word-scale visualizations exist, interactive word-scale visualizations are still a new concept. Moreover, we still know little about the effect of introducing interactive versions of these charts in online reading environments. Therefore, we ran two experiments to investigate participants' experience using our active reading prototype. We focused on first studying a small set of primary interaction techniques that make it possible to create and explore small multiples overviews and compared these against using statically embedded word-scale visualizations. In our second experiment, we gave participants a broader set of interaction techniques and allowed them to freely explore 3 articles and answer an open-ended question.

\section{Participants}

For the two experiments, we recruited 6 female and 6 male participants with ages ranging from $20-30$ years ( 9 participants), 31-40 (1), and 41-50 (2). Their occupations included: 

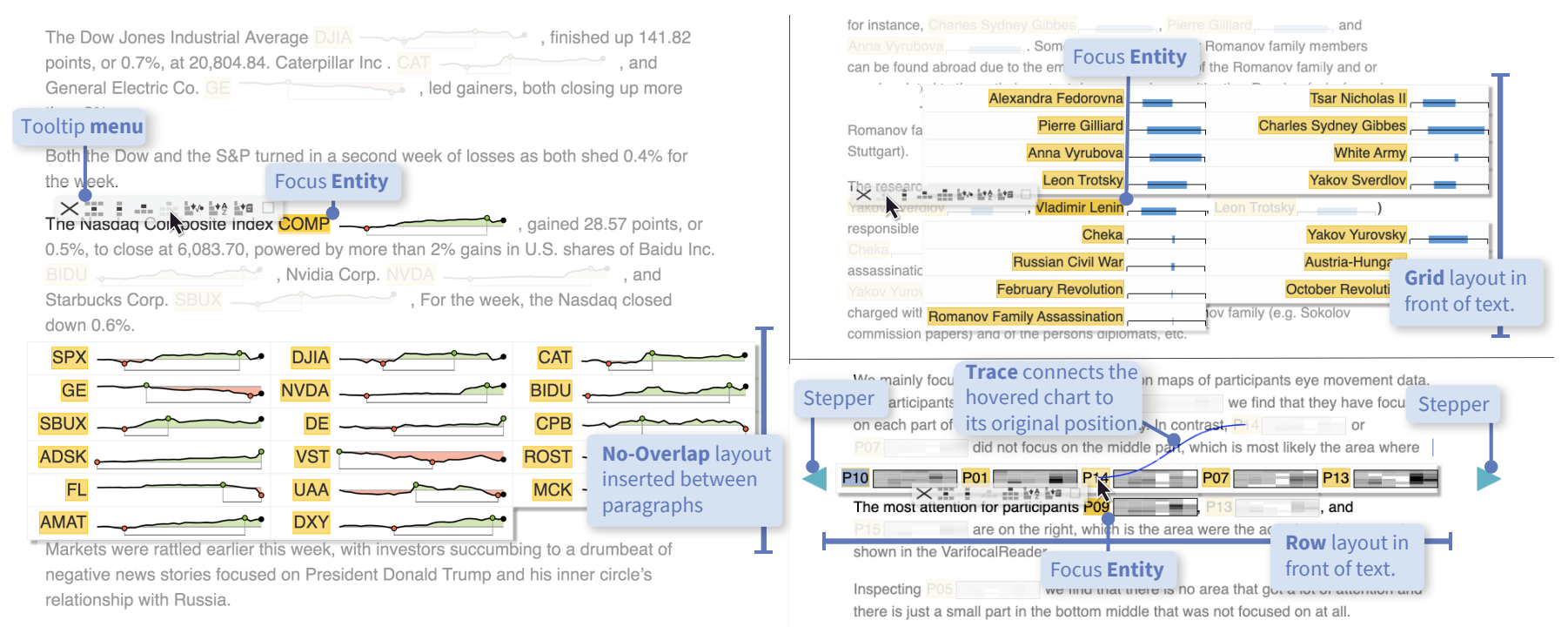

Figure 3. Our economic news prototype (left) showing stock charts in a no-overlap grid. Our European history prototype (top right) showing timelines in an in-front grid. (bottom right) Our eye-tracking report prototype with attention maps in a row layout. In all three, charts are grouped around a focus entity (COMP, Vladimir Lenin, and P09). Left and right steppers on the row layout jump back and forth through the full set of attention maps.

3 undergraduates, $4 \mathrm{PhD}$ researchers, 2 postdocs, 1 designer, 1 engineer, and 1 researcher. Participants reported expertise in the following areas: visualization and/or HCI (8), computer science (2), topology (1), and publications/web design (1).

\section{Experiment 1: Interactive versus Static Gathering}

In Experiment 1, we tested the impact of interaction techniques for word-scale visualizations on search and comparison tasks. For simplicity, we included only a small set of core interactions and one layout, allowing participants to gather word-scale visualizations into a grid layout, sort them, and compare them using brushing and linking.

In each trial, participants examined a short news article (7561368 words, $\mathrm{SD}=132$ ) from MarketWatch [1], which included word-scale charts associated with each of the 16-27 $(\mathrm{SD}=3)$ stocks or indexes mentioned in the text. Inspired by Bertin [14, 15] we chose two types of tasks for the experiment: search tasks, during which participants located and extracted information from a single word-scale visualization, and more complex comparison tasks, which required them to visually scan all of the word-scale visualizations and identify a single outlier. Examples of each prompt are shown below:

Search. "Did the stock price for $\boldsymbol{L U V}$ spend the majority of the previous month above or below its starting value?"

Compare. "Over the whole period, which stock had the smallest number of days between its highest and lowest points?"

For both types of tasks we compared participants' performance across two interface conditions:

Static. Participants could scroll through the article, but could not gather or sort word-scale visualizations.

Interactive. Participants could gather all word-scale visualizations into the grid layout, sort them by position in the document, by last value, or by entity name.
To help participants make accurate judgments about the content of the visualizations, we enabled brushing and linking interactions in both conditions.

Participants entered their response using a drop-down or text area located to the right of the document. We measured participants' task completion time and error rate for each trial. In addition, we logged all of their interactions with the document or visualizations and tracked their total scroll distance. See supplemental material for more details on this data.

\section{Experiment 2: Open-Ended Exploration Task}

In the second experiment, we wanted to examine how participants might use a broader set of view manipulation interactions to solve an open-ended analysis task. We gave participants a longer set of 3 MarketWatch articles from mid-2017. Two articles included 27 stock charts while the third included 20. Using these documents we gave participants an open-ended question that encouraged them to make a decision based on both the text and the word-scale visualizations:

Exploration Task. "Imagine that it is August $4^{\text {th }}, 2017$ and you are preparing to make some new investments. Using the information in the 3 provided articles, list the top 3-5 stocks or indexes you would consider investing in and briefly explain why you would choose each."

For this task, we allowed participants to use all of the gathering, sorting, and brushing and linking interactions from Experiment 1. Participants were also able to use additional layouts (grid, column, and row), placement options (in-front and no-overlap), and alignments (column-aligned and row pan-aligned). We also enabled the document navigation interaction, which allowed participants to navigate from gathered word-scale visualizations back to their original positions in the text. Additionally, we added a simple hovering interaction, that allowed participants to see where in the document a wordscale visualization came from. As in Experiment 1, we logged all interactions with the documents and visualizations. 


\section{Apparatus and Procedure}

We conducted the study in a room with three workstations, which allowed us to run multiple participants simultaneously. Each participant used an HP Z800 PC with Windows 10, a Dell 20" monitor $(1600 \times 900 \mathrm{px})$, and Google Chrome.

After participants arrived, they selected a numeric participant ID and signed a consent form. Participants then selfadministered the study using a set of on-screen instructions. During the first experiment, each participant completed the same four blocks of tasks, starting with two blocks of search tasks, then completing two blocks of comparison tasks. We counterbalanced the static vs. interactive condition based on the parity of the participant ID. At the beginning of each block, participants read a set of general instructions and completed a tutorial to familiarize themselves with the current task and interface. Participants then completed 3 training trials and 16 trials in each of the four blocks. In total this amounted to 76 trials $(2$ conditions $\times 2$ question types $\times(3$ training + 16 trials)). Participants could repeat the 3 training trials until they felt comfortable. We instructed participants to answer the questions as quickly and accurately as possible. After completing all trials, the system directed participants to Google forms for a follow-up questionnaire (see supplemental material).

For Experiment 2, participants again read general instructions and completed a tutorial that explained each of the available interactions and gave opportunity to try them. The system then instructed participants to spend 15 minutes exploring three different articles and crafting their response. We showed a timer for the duration of their exploration and a pop-up message after the time had elapsed, encouraged participants to finish up. Finally, participants completed a second questionnaire (see supplemental material).

\section{Data Analysis}

For Experiment 1, we performed an initial analysis of time and error rates by condition (static/interactive) separately for each task type (search/compare) according to a pre-registered procedure $^{1}$. We report means and $95 \%$ confidence intervals and use interval estimation rather than statistical significance tests to interpret our results [26].

We screened participants to exclude any whose average task completion times or error rates were more than 3 standard deviations from the mean across all participants. No participants met this threshold and we retained data from all 12 participants. In our initial analysis of task completion times, we also discarded data for two trials which included long gaps during which no interactions or scrolling occurred (59.4 seconds for one of P6's trials, and 118.4 seconds for one of P5's). Analysis workbooks are included as supplementary material and posted publicly with our pre-registration ${ }^{1}$.

\section{Secondary Analysis}

In addition to the planned time and error analyses, we also analyzed the difference in scroll distance by task and condition. Moreover, we conducted exploratory analyses of the questionnaires from Experiment 1 as well as the logs and

\footnotetext{
${ }^{1}$ https://osf.io/4btpx/?view_only=a89c58e8bc154fdda09d26e7d3face7f
}
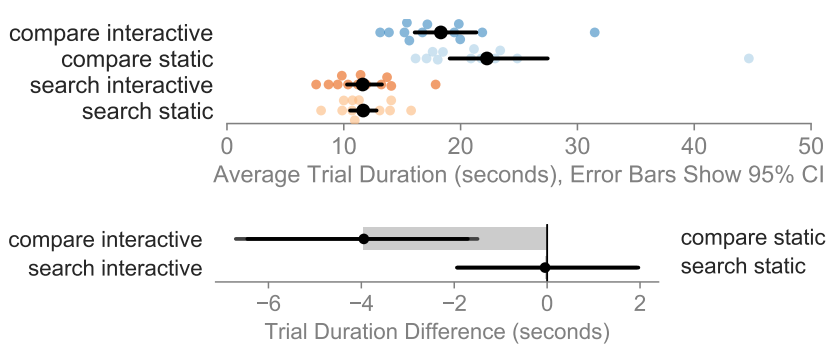

Figure 4. Average per-participant task times (top) and pairwise differences (bottom) for Experiment 1 with means and $95 \%$ bootstrap CIs.

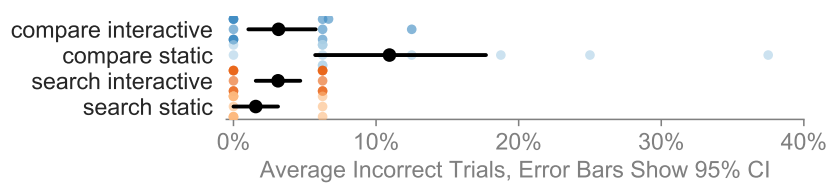

Figure 5. Average per-participant error for Experiment 1 with means and $95 \%$ bootstrap CIs.

questionnaires from Experiment 2. To analyze open-ended responses, one of the authors coded them then discussed and iteratively analyzed them with the other co-authors.

\section{RESULTS}

Participants took on average $86 \mathrm{~min}(\mathrm{SD}=14)$ to complete the study, with the average participant completing Experiment 1 in 57.7 min $(\mathrm{SD}=10.8)$ and Experiment 2 in $28.7 \mathrm{~min}(\mathrm{SD}=7.4)$ including time used to answer the questionnaires.

\section{Experiment 1}

Overall, the analysis showed little variation in performance between the two conditions for the simpler search tasks. However, the interaction techniques helped participants perform comparison tasks faster and more accurately, while also scrolling considerably less.

Time, Error, and Scrolling

For search tasks, we saw no evidence for a difference in speed between the static $(\mathrm{M}=11.6 \mathrm{~s}, \mathrm{CI}=[10.5 \mathrm{~s}, 12.8 \mathrm{~s}])$ and the interactive condition $(M=11.6 \mathrm{~s}, \mathrm{CI}=[10.2 \mathrm{~s}, 13.2 \mathrm{~s}]$, Figure 4$)$. Similarly, in search tasks participants made a comparable number of errors in both the static $(\mathrm{M}=1.6 \%, \mathrm{CI}=[0.0 \%, 3.1 \%])$ and interactive $(\mathrm{M}=3.1 \%, \mathrm{CI}=[1.5 \%, 4.7 \%]$, Figure 5) conditions.

However, for the more involved comparison tasks, participants were faster in the interactive condition $(M=18.2 \mathrm{~s}$, $\mathrm{CI}=[16.0 \mathrm{~s}, 21.3 \mathrm{~s}])$ than the static $(\mathrm{M}=22.1 \mathrm{~s}, \mathrm{CI}=[19.1 \mathrm{~s}, 27.0 \mathrm{~s}])$. Participants also made fewer errors in the interactive condition $(\mathrm{M}=3.2 \%, \mathrm{CI}=[1.0 \%, 5.7 \%])$ than in the static condition $(\mathrm{M}=10.9 \%, \mathrm{CI}=[5.7 \%, 17.7 \%])$.

Our post-hoc analysis revealed dramatic differences in the amount of scrolling between the two conditions (Figure 6). During search tasks, participants scrolled an average of 1956 pixels $(\mathrm{CI}=[1801,2184])$ in the static condition, approximately four times as far as in the interactive condition ( $M=472$ pixels, $\mathrm{CI}=[75,1060])$. This difference was even more pronounced for comparison tasks, with participants scrolling roughly 80 times as far in the static condition (M=3894 pixels, $\mathrm{CI}=[3271,4732])$ as in the interactive condition ( $M=48$ pixels, $\mathrm{CI}=[7,109])$. 

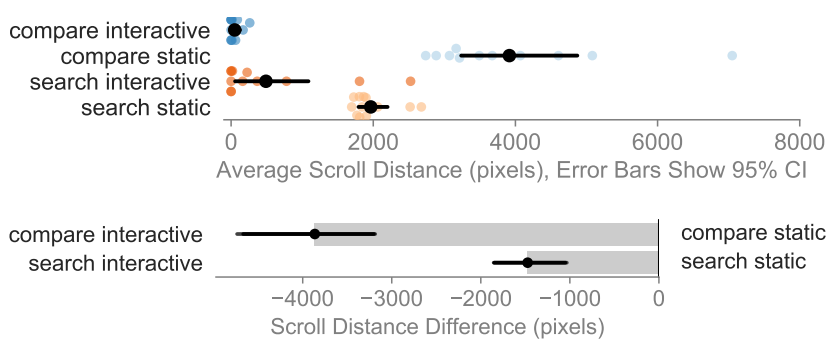

Figure 6. Average per-participant scroll distances (top) and pairwise differences (bottom) for Experiment 1 with means and 95\% bootstrap CIs.

\begin{tabular}{c|cccc|c} 
& \multicolumn{4}{|c|}{ Experiment 1 } & 2 \\
Approach & Compare & Search & \\
& Interac. & Static & Interac. & Static & \\
\hline Skim or Read Document & & 6 & 1 & 5 & 8 \\
Create Overview & 10 & & 10 & 0 & 7 \\
Visually Estimate & 8 & & 6 & 9 & 11 \\
Brush & 3 & & 3 & 9 & 2 \\
Reorder & & & 6 & & \\
Compare Across Articles & - & - & - & & 5
\end{tabular}

Figure 7. Number of participants who reported using various approaches during the studies (by experiment, task, and condition).

\section{Strategies}

Participants reported using a number of different approaches (Figure 7) to complete the tasks in Experiment 1. When performing both types of tasks using static word-scale visualizations, participant mainly skimmed the document and visually estimated the area or line length, using brushing to count the exact number of days if necessary. In the interactive condition, participants still visually estimated area or line length and used brushing to count, but did so almost exclusively using the collected grid of charts, rather than by skimming.

\section{Summary}

Participants found both the static and the interactive wordscale visualization helpful and useful for the given tasks. They chose to use the interactive features when they were available and appreciated the ease of comparison and reduced need for scrolling. Participants' perceived reduction in task completion time was further confirmed by the quantitative results for the comparison task. We also collected suggestions from participants which included requests for faster gathering animations, showing arrangements next to the cursor, alternative grid arrangements and sorting options, as well as the ability to annotate word-scale visualizations.

\section{Experiment 2}

Participants spent an average of 18.7 minutes $(\mathrm{SD}=5.1)$ in total on the task, exploring the 3 articles using a broader range of view manipulation interactions.

\section{Interaction Logs and Task Completion Actions}

Analyzing the interaction logs we see that all participants except P07 used gathering to perform a visualization-centric analysis of the texts (Figure 8). Some participants also conducted more in-depth analyses applying brushing and linking, hovering, sorting, or layout changing interactions. At the end

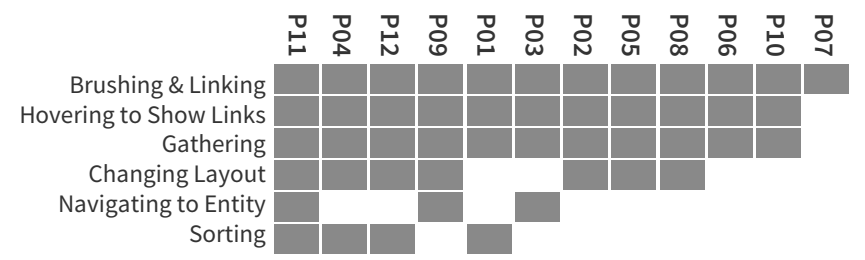

Figure 8. Interaction techniques used in Experiment 2.

of the study participants described the approaches they used to answer the question (Figure 7).

Overall, the combination of interaction logs analysis and the reported strategies suggests that most participants used both document-centric as well as visualization-centric approaches, leveraging multiple interaction techniques to do so.

\section{Summary}

Our log analysis of Exp 2 shows that participants made good use of the available interactive options (Figure 8), using a variety of actions. Yet, participants used a somewhat different mix of interactions than in Experiment 1, which is not surprising given the more exploratory prompt. Despite training, participants made little use of sorting and navigation in particular. Interestingly, most participants used the grid layout that was provided in Experiment 1 and only four participants tried the other grid options at all. The improvements and feedback we received for Experiment 2 were similar to those we collected for Experiment 1 and were mainly concerned with the positioning of the grid as well as the slow animation speed.

\section{DISCUSSION}

Overall, our prototype implementation highlights one possible approach for integrating the key interactions from our design space. Below we discuss further options to broaden our design space and elaborate on how interactive word-scale visualizations fit into the broader space of document-centric and visualization-centric analysis.

\section{Broadening the Design Space}

The design space we explored focuses primarily on interaction techniques that support what Heer and Shneiderman [35] call "view manipulations." Yet, the layouts and arrangements created with our interaction techniques also serve as a starting point for tasks in their "data \& view specification" category (which includes activities such as filtering or sorting) and their "process and provenance" category (with activities such as annotation or sharing for collaboration). For example, gathered overviews simplify the task of filtering and sharing these overviews can aid collaboration.

Our current design space includes several interaction techniques that are useful for comparing groups of word-scale visualizations but we have not yet deeply explored operations that filter based on the displayed data or that change encoding properties of word-scale visualizations-which Yi et al. [62] also identify as important for visualization. One possible way of supporting operations like filtering is to treat word-scale visualizations as tokens that can be dynamically manipulated, shifted, and organized into groups, stacks, and piles. Using 
physically-inspired and force-directed interactions like those in "Kinetica" [48] or piling and sorting techniques like in "Small MultiPiles" [5] could enable more nuanced and creative ways to explore data embedded in documents or even manipulate the document itself.

The techniques we implemented are mostly initiated from the menu or from a single word-scale visualization. However, these long horizontal menus are limiting and can hide important text, making alternatives such as pie menus [22] an appealing choice. Another promising interaction technique is Crossets [46] which could transform interactions like sorting into direct manipulations using the word-scale visualizations themselves. However, these kinds of techniques call for wordscale visualization designs which strongly suggest to readers that these interactions are possible $[17,18]$.

Finally, our prototypes examine view manipulation interactions with text documents including economic news articles, history texts, and research reports. However, word-scale visualizations in interactive applications like Hoffswell et al.'s [36] code editor present new opportunities and challenges. In these contexts, both visualizations and text may appear and disappear dynamically. Moreover, applications for source code and larger document corpora may need to gather or connect word-scale visualizations from across multiple documents.

\section{Document-Centric to Visualization-Centric Analysis}

In Experiment 2 we showed how view manipulation interactions can help bridge the gap between document-centric analysis - in which the text is the focus of attention-and visualization-centric analysis-in which visual representations of data derived from the text are the focus [34]. Fully document-centric text analysis tools display the text itself, often with simple highlighting or annotations added. Meanwhile, visualization-centric approaches, such as PhraseNets [58] or Wordle [60] focus almost exclusively on the text as data, displaying entities and their relationships divorced from the original documents. In the middle are hybrid techniques such as Jigsaw [50], DocuBurst [25], or Elastic Documents [6] that either offer side-by-side views of visualizations and original text or data, or allow a reader to switch between reading visualizations and source texts.

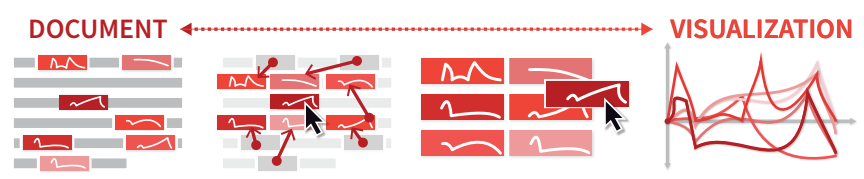

Figure 9. From left to right: Document with embedded word-scale visualizations; word-scale visualizations collected around an entity of interest with text visible; small multiples without text; single visualization.

Helping readers transition from document-centric views to visualization-centric ones and back during the course of an analysis may lead to more effective analyses which incorportate both analytical findings from the data and context from the documents. Our view manipulation interactions aid transitions between both types of views by introducing a number of interesting intermediate stages (Figure 9). A document with word-scale visualizations represents one step beyond a purely document-centric view. While the text remains the focus, the embedded visualizations reveal additional data incontext. Small multiples visualizations created by using a gathering interaction represent a pivot point along this spectrum and may serve as a transition between document- and visualization-centric modes. If the data is of greater interest to readers, they may then hide the text completely, allowing the small multiples layout to transform into a more general purpose text analysis environment, as in Van den Elzen's and Van Wijk's "Small Multiples, Large Singles" [57]. However, when the text again becomes important, this process could be reversed, fluidly re-introducing the visualizations back into the document. As such, interactive word-scale visualizations can also let readers shift between author-driven and readerdriven narrative modes as noted in the data storytelling literature [49]. Author-driven analyses require a set reading order while reader-driven analyses allow free exploration of the data.

\section{Word-Scale Visualizations and Readability}

While our results highlight the potential of interactive view manipulations for word-scale visualizations, additional research is needed to understand their impact on document readability. For example, our prototypes all embedded visualizations inline in sentences, which could alter reading behavior. However, a wide variety of other placement options [33] — including positioning visualizations in the margins or in tooltips-are also compatible with our proposed interactions. More detailed eye-tracking and readability studies could help characterize the trade-offs between these approaches.

\section{CONCLUSION}

Our design space of view manipulation interactions for wordscale visualizations introduces a variety of new and promising techniques for collecting, arranging, comparing, and navigating small visualizations embedded across larger documents. Building on this, our interactive prototypes highlight the utility of these techniques for documents and datasets from a variety of domains including economic news, European history, and eye-tracking research. Interactive word-scale visualizations allow readers to see more detail and to make comparisons between visualizations in-context with document text. This allows readers the flexibility to examine documents and data together in ways that are not possible in most current reading environments. Study participants were able to complete comparison tasks across multiple word-scale visualizations faster and with fewer errors. Qualitative feedback was also positive, providing evidence that the added flexibility did not have a negative impact on people's ability to read the text and explore the associated data. While the space of view manipulation interactions for word-scale visualizations has been largely unexplored, we provide a first step towards a more comprehensive understanding of these techniques. Moving forward, we hope that these interactions can serve as a basis for new systems that allow even richer integration between text and visualizations.

\section{ACKNOWLEDGMENTS}

This work was sponsored by the French Research Organization, project grant ANR-11-JS02-003 and supported by the Collaborative European Digital Archive Infrastructure project CENDARI as well as a France-Canada mobility grant. 


\section{REFERENCES}

[1] Last read: March 2019. MarketWatch. (Last read: March 2019). https://www.marketwatch.com.

[2] Last read: September 2019. Dictionary.com. (Last read: September 2019). https://www. dictionary.com.

[3] Eytan Adar, Daniel S Weld, Brian N Bershad, and Steven S Gribble. 2007. Why we search: visualizing and predicting user behavior. In Proceedings of the 16th International Conference on World Wide Web. ACM, 161-170. DOI :

http://dx.doi .org/10.1145/1242572.1242595

[4] Benjamin Bach, Nathalie Henry Riche, Sheelagh Carpendale, and Hanspeter Pfister. 2017. The emerging genre of data comics. Computer Graphics and Applications 37, 3 (2017), 6-13. DOI:

http://dx.doi.org/10.1109/MCG.2017.33

[5] Benjamin Bach, Nathalie Henry Riche, Tim Dwyer, Tara Madhayastha, Jean-Daniel Fekete, and Thomas Grabowski. 2015. Small MultiPiles: Piling time to explore temporal patterns in dynamic networks. Computer Graphics Forum 34, 3 (2015), 31-40. DOI : http://dx.doi.org/10.1111/cgf.12615

[6] Sriram Karthik Badam, Zhicheng Liu, and Niklas Elmqvist. 2019. Elastic documents: Coupling text and tables through contextual visualizations for enhanced document reading. IEEE Transactions on Visualization and Computer Graphics 25, 1 (2019), 661-671. DOI : http://dx.doi.org/10.1109/TVCG.2018.2865119

[7] Sebastian Baltes, Oliver Moseler, Fabian Beck, and Stephan Diehl. 2015. Navigate, understand, communicate: How developers locate performance bugs. In ACM/IEEE International Symposium on Empirical Software Engineering and Measurement (ESEM). IEEE, 1-10. DOI :

http://dx.doi.org/10.1109/ESEM. 2015.7321208

[8] Fabian Beck, Yasett Acurana, Tanja Blascheck, Rudolf Netzel, and Daniel Weiskopf. 2016. An expert evaluation of word-sized visualizations for analyzing eye movement data. In IEEE Second Workshop on Eye Tracking and Visualization (ETVIS). IEEE, 50-54. DOI : http://dx.doi.org/10.1109/ETVIS.2016.7851166

[9] Fabian Beck, Tanja Blascheck, Thomas Ertl, and Daniel Weiskopf. 2016. Word-sized eye tracking visualizations. In Burch M., Chuang L., Fisher B., Schmidt A., Weiskopf D. (eds) Eye Tracking and Visualization. ETVIS 2015. Springer. DOI :

http://dx.doi .org/10.1007/978-3-319-47024-5_7

[10] Fabian Beck, Sebastian Koch, and Daniel Weiskopf. 2015. Visual analysis and dissemination of scientific literature collections with SurVis. IEEE Transactions on Visualization and Computer Graphics 22, 1 (2015), 180-189. DOI :

http://dx.doi.org/10.1109/TVCG.2015.2467757
[11] Fabian Beck, Oliver Moseler, Stephan Diehl, and Günter Daniel Rey. 2013. In situ understanding of performance bottlenecks through visually augmented code. In 21 st International Conference on Program Comprehension (ICPC). IEEE, 63-72. DOI : http://dx.doi.org/10.1109/ICPC. 2013.6613834

[12] Fabian Beck and Daniel Weiskopf. 2017. Word-sized graphics for scientific texts. IEEE Transactions on Visualization and Computer Graphics 23, 6 (2017), 1576-1587. DOI :

http://dx.doi.org/10.1109/TVCG. 2017.2674958

[13] Richard A Becker and William S Cleveland. 1987. Brushing scatterplots. Technometrics 29, 2 (1987), 127-142. DOI : http://dx . doi .org/10.2307/1269768

[14] Jacques Bertin. 1967. Sémiologie graphique: Les diagrammes-Les réseaux-Les cartes. Mouton/Gauthier-Villars.

[15] Jacques Bertin. 1983. Semiology of Graphics: Diagrams, Networks, Maps. University of Wisconsin Press, Madison, WI, USA.

[16] Anastasia Bezerianos, Fanny Chevalier, Pierre Dragicevic, Niklas Elmqvist, and Jean-Daniel Fekete. 2010. GraphDice: A system for exploring multivariate social networks. Computer Graphics Forum 29, 3 (2010), 863-872. DOI : http://dx.doi.org/10.1111/j.1467-8659.2009.01687.x

[17] Tanja Blascheck, Lindsay MacDonald Vermeulen, Jo Vermeulen, Charles Perin, Wesley Willett, Thomas Ertl, and Sheelagh Carpendale. 2019. Exploration strategies for discovery of interactivity in visualizations. IEEE Transactions on Visualization and Computer Graphics 25, 2 (2019), 1407-1420. DOI : http://dx.doi.org/10.1109/TVCG. 2018.2802520

[18] Jeremy Boy, Louis Eveillard, Françoise Detienne, and Jean-Daniel Fekete. 2016. Suggested Interactivity: Seeking Perceived Affordances for Information Visualization. IEEE Transactions on Visualization and Computer Graphics 22, 1 (2016), 10. DOI : http://dx.doi.org/10.1109/TVCG. 2015.2467201 To appear.

[19] Ulrik Brandes, Bobo Nick, Brigitte Rockstroh, and Astrid Steffen. 2013. Gestaltlines. Computer Graphics Forum 32, 3 (2013), 171-180. DOI : http://dx.doi.org/10.1111/cgf.12104

[20] Richard Brath, Peter MacMurchy, and Ebad Banissi. 2019. The design space of SparkWords. In EuroVis 2019 - Short Papers. The Eurographics Association, 127-131. DOI : http://dx.doi.org/10.2312/evs. 20191182

[21] Aaron Bycoffe. Last read: September 2019. The Endorsement Primary. (Last read: September 2019). https://projects.fivethirtyeight.com/ 2016-endorsement-primary/.

[22] Jack Callahan, Don Hopkins, Mark Weiser, and Ben Shneiderman. 1988. An empirical comparison of pie vs. 
linear menus. In Proceedings of the SIGCHI Conference on Human Factors in Computing Systems (CHI). ACM, 95-100. DOI : http://dx.doi.org/10.1145/57167.57182

[23] Bay-Wei Chang, Jock Mackinlay, and Polle T Zellweger. 2000. Fluidly revealing information in fluid documents. In Proceedings of Smart Graphics. AAAI, Menlo Park, CA, USA, 178-181.

[24] John B Cole and Paul M VanRaden. 2010. Visualization of results from genomic evaluations. Journal of Dairy Science 93, 6 (2010), 2727-2740. DOI:

http://dx.doi.org/10.3168/jds.2009-2763

[25] Christopher Collins, Sheelagh Carpendale, and Gerald Penn. 2009. Docuburst: Visualizing document content using language structure. Computer Graphics Forum 28, 3 (2009), 1039-1046. DOI :

http://dx.doi.org/10.1111/j.1467-8659.2009.01439.x

[26] Pierre Dragicevic. 2016. Fair statistical communication in HCI. In Modern Statistical Methods for HCI.

Springer, 291-330. DOI :

http://dx.doi.org/10.1007/978-3-319-26633-6_13

[27] Pierre Dragicevic, Yvonne Jansen, Abhraneel Sarma, Matthew Kay, and Fanny Chevalier. 2019. Increasing the transparency of research papers with explorable multiverse analyses. In Proceedings of the SIGCHI Conference on Human Factors in Computing Systems (CHI). ACM. DOI :

http://dx.doi.org/10.1145/3290605.3300295

[28] Niklas Elmqvist, Andrew Vande Moere, Hans-Christian Jetter, Daniel Cernea, Harald Reiterer, and TJ Jankun-Kelly. 2011. Fluid interaction for information visualization. Information Visualization 10, 4 (2011), 327-340. DOI :

http://dx.doi.org/10.1177/1473871611413180

[29] Leo D Frishberg. 2011. Interactive sparklines: A dynamic display of quantitative information. In Extended Abstracts on Human Factors in Computing Systems (CHI). ACM, 589-604. DOI :

http://dx.doi.org/10.1145/1979742.1979655

[30] Sohaib Ghani, Nathalie Henry Riche, and Niklas Elmqvist. 2011. Dynamic insets for context-aware graph navigation. Computer Graphics Forum 30, 3 (2011), 861-870. DOI :

http://dx.doi.org/10.1111/j.1467-8659.2011.01935.x

[31] Michael Gleicher, Danielle Albers, Rick Walker, Ilir Jusufi, Charles D Hansen, and Jonathan C Roberts. 2011. Visual comparison for information visualization. Information Visualization 10, 4 (2011), 289-309. DOI: http://dx.doi.org/10.1177/1473871611416549

[32] Pascal Goffin, Jeremy Boy, Wesley Willett, and Petra Isenberg. 2016. An exploratory study of word-scale graphics in data-rich text documents. IEEE Transactions on Visualization and Computer Graphics 23, 10 (2016), 2275-2287. DOI :

http://dx.doi.org/10.1109/TVCG.2016.2618797
[33] Pascal Goffin, Wesley Willett, Jean-Daniel Fekete, and Petra Isenberg. 2014. Exploring the placement and design of word-scale visualizations. IEEE Transactions on Visualization and Computer Graphics 20, 12 (2014), 2291-2300. DOI :

http://dx.doi.org/10.1109/TVCG. 2014.2346435

[34] Pascal Goffin, Wesley Willett, Jean-Daniel Fekete, and Petra Isenberg. 2015. Design considerations for Enhancing Word-Scale Visualizations with Interaction. In Posters of the IEEE Conference on Information Visualization (InfoVis). IEEE, Los Alamitos, CA, USA.

[35] Jeffrey Heer and Ben Shneiderman. 2012. Interactive Dynamics for Visual Analysis. Communications of the ACM 55, 4 (2012), 45-54. DOI : http://dx.doi.org/10.1145/2133806.2133821

[36] Jane Hoffswell, Arvind Satyanarayan, and Jeffrey Heer. 2018. Augmenting code with in situ visualizations to aid program understanding. In Proceedings of the SIGCHI Conference on Human Factors in Computing Systems (CHI). ACM, 532. DOI :

http://dx.doi.org/10.1145/3173574.3174106

[37] Steffen Koch, Michael John, Michael Wörner, Andreas Müller, and Thomas Ertl. 2014. VarifocalReader in-depth visual analysis of large text documents. IEEE Transactions on Visualization and Computer Graphics 20, 12 (2014), 1723-1732. DOI : http: //dx. doi .org/10.1109/TVCG. 2014.2346677

[38] Nicholas Kong, Marti A Hearst, and Maneesh Agrawala. 2014. Extracting references between text and charts via crowdsourcing. In Proceedings of the SIGCHI Conference on Human Factors in Computing Systems (CHI). ACM, 31-40. DOI : http://dx.doi .org/10.1145/2556288.2557241

[39] Shahid Latif and Fabian Beck. 2018. Visually augmenting documents with data. Computing in Science Engineering 20, 6 (2018), 96-103. DOI : http://dx.doi.org/10.1109/MCSE. 2018.2875316

[40] Shahid Latif and Fabian Beck. 2019. VIS author profiles: Interactive descriptions of publication records combining text and visualization. IEEE Transactions on Visualization and Computer Graphics 25, 1 (2019), 152-161. DOI : http://dx.doi.org/10.1109/TVCG. 2018.2865022

[41] Shahid Latif, Diao Liu, and Fabian Beck. 2018. Exploring interactive linking between text and visualization. In EuroVis 2018 - Short Papers. Eurographics Association, 91-94. D0I : http://dx.doi .org/10.2312/eurovisshort. 20181084

[42] Tomer Moscovich, Fanny Chevalier, Nathalie Henry Riche, Emmanuel Pietriga, and Jean-Daniel Fekete. 2009. Topology-Aware Navigation in Large Networks. In Proceedings of the SIGCHI Conference on Human Factors in Computing Systems (CHI). ACM, 2319-2328. DOI :

http://dx.doi.org/10.1145/1518701.1519056 
[43] Daniela Oelke, David Spretke, Andreas Stoffel, and Daniel A Keim. 2012. Visual readability analysis: How to make your writings easier to read. IEEE Transactions on Visualization and Computer Graphics 18, 5 (2012), 662-674. DOI :

http://dx.doi.org/10.1109/TVCG.2011.266

[44] Jonas Parnow. 2015. Micro Visualizations: How can Micro Visualisations enhance text comprehension, memorability, and exploitation? Design Master Thesis. Potsdam University of Applied Sciences, Potsdam, Germany. http://www.microvis.info.

[45] Jonas Parnow and Marian Dörk. 2015. Micro Visualizations: Data-driven typography and graphical text enhancement. In Posters of the Conference on Information Visualization (InfoVis). IEEE.

[46] Charles Perin, Pierre Dragicevic, and Jean-Daniel Fekete. 2015. Crossets: manipulating multiple sliders by crossing. In Proceedings of the 41st Graphics Interface Conference. Canadian Information Processing Society, 233-240. DOI :

http://dx.doi .org/10.1145/2670444.2670449

[47] Charles Perin, Romain Vuillemot, and Jean-Daniel Fekete. 2013. SoccerStories: A kick-off for visual soccer analysis. IEEE Transactions on Visualization and Computer Graphics 19, 12 (2013), 2506-2515. DOI : http://dx. doi.org/10.1109/TVCG. 2013.192

[48] Jeffrey M Rzeszotarski and Aniket Kittur. 2014. Kinetica: naturalistic multi-touch data visualization. In Proceedings of the SIGCHI Conference on Human Factors in Computing Systems (CHI). ACM, 897-906. DOI:http://dx.doi.org/10.1145/2556288.2557231

[49] Edward Segel and Jeffrey Heer. 2010. Narrative visualization: Telling stories with data. IEEE Transactions on Visualization and Computer Graphics 16, 6 (2010), 1139-1148. DOI :

http://dx.doi.org/10.1109/TVCG. 2010.179

[50] John Stasko, Carsten Gorg, Zhicheng Liu, and Kanupriya Singhal. 2007. Jigsaw: Supporting investigative analysis through interactive visualization. In Proceedings of the IEEE Symposium on Visual Analytics Science and Technology (VAST). IEEE, 131-138. DOI :

http://dx.doi.org/10.1109/VAST. 2007.4389006

[51] Markus Steinberger, Manuela Waldner, Marc Streit, Alexander Lex, and Dieter Schmalstieg. 2011. Context-preserving visual links. IEEE Transactions on Visualization and Computer Graphics 17, 12 (2011), 2249-2258. DOI :

http://dx.doi.org/10.1109/TVCG.2011.183

[52] Bongwon Suh, Allison Woodruff, Ruth Rosenholtz, and Alyssa Glass. 2002. Popout Prism: Adding perceptual principles to overview+detail document interfaces. In Proceedings of the SIGCHI Conference on Human Factors in Computing Systems (CHI). ACM, 251-258. DOI: http://dx.doi.org/10.1145/503376.503422

[53] Ben Swift, Andrew Sorensen, Henry Gardner, and John Hosking. 2013. Visual code annotations for cyberphysical programming. In 1st International Workshop on Live Programming (LIVE). IEEE, 27-30. DOI : http://dx.doi.org/10.1109/LIVE.2013.6617345

[54] Christian Tominski, Camilla Forsell, and Jimmy Johansson. 2012. Interaction support for visual comparison inspired by natural behavior. IEEE Transactions on Visualization and Computer Graphics 18, 12 (2012), 2719-2728. DOI :

http://dx. doi .org/10.1109/TVCG. 2012.237

[55] Edward R. Tufte. 2001. The visual display of quantitative information. Graphics Press, Cheshire, CT.

[56] Edward R. Tufte. 2006. Beautiful evidence. Graphics Press, Cheshire, CT.

[57] Stef van den Elzen and Jarke J van Wijk. 2013. Small multiples, large singles: A new approach for visual data exploration. Computer Graphics Forum 32, 3pt2 (2013), 191-200. DOI: http://dx.doi.org/10.1111/cgf.12106

[58] Frank Van Ham, Martin Wattenberg, and Fernanda B Viégas. 2009. Mapping text with phrase nets. IEEE Transactions on Visualization and Computer Graphics 15, 6 (2009), 1169-1176. DOI :

http://dx. doi .org/10.1109/TVCG. 2009.165

[59] Bret Victor. Last read: March 2019. Explorable explanation. (Last read: March 2019). http://worrydream. com/ExplorableExplanations/.

[60] Fernanda B Viegas, Martin Wattenberg, and Jonathan Feinberg. 2009. Participatory visualization with wordle. IEEE Transactions on Visualization and Computer Graphics 15, 6 (2009), 1137-1144. DOI:

http://dx. doi .org/10.1109/TVCG. 2009.171

[61] Gareth Watts. Last read: March 2019. jQuery Sparklines. (Last read: March 2019).

https://omnipotent.net/jquery.sparkline/.

[62] Ji Soo Yi, Youn ah Kang, John T Stasko, and Julie A Jacko. 2007. Toward a deeper understanding of the role of interaction in information visualization. IEEE Transactions on Visualization and Computer Graphics 13, 6 (2007), 1224-1231. DOI :

http://dx.doi.org/10.1109/TVCG.2007.70515

[63] Dongwook Yoon, Nicholas Chen, and François Guimbretière. 2013. TextTearing: Opening white space for digital ink annotation. In Proceedings of the Conference on User Interface Software and Technology (UIST). ACM, 107-112. DOI :

http://dx.doi.org/10.1145/2501988.2502036

[64] Polle Zellweger, Bay-Wei Chang, and Jock D Mackinlay. 1998. Fluid Links for Informed and Incremental Link Transitions. In Proceedings of the Ninth ACM Conference on Hypertext and Hypermedia: Links, Objects, Time and Space-Structure in Hypermedia Systems: Links, Objects, Time and Space-Structure in Hypermedia Systems, Vol. 98. ACM, 50-57. DOI : http://dx.doi.org/10.1145/276627.276633 